\section{WEB WATCH}

\section{Model resource}

- http://www.ebi.ac.uk biomodels

A step towards the greater use of computational models in biology was taken when the world's first database of annotated biological models went online on 11 April 2005.

The Biomodels Database has been designed to allow biologists to store, search and retrieve published mathematical models of biological systems. The

free-to-use database aims to provide access to published, peer-reviewed, quantitative models of biochemical and cellular systems.

So far, there's been no 'official' way for biologists to readily share such models, but they can now produce and freely distribute their models using the widely accepted, open-source Systems Biology Markup Language (SBML). Once entered into the database, models are annotated and linked to relevant data sources, such as publications or other databases, by human curators.

The database is the result of a collaboration led by the European Bioinformatics Institute (part of the

European Molecular Biology Laboratory) in the United Kingdom and the SBML team from the United States. Other contributors come from the United States, Japan and South Africa.

The developers' hope is that, ultimately, scientists will be encouraged to deposit their published models in the Biomodels Database, making them freely available to all.

The database is currently open to the submission of models - the curators will then pick them up for syntax and semantic curation and submit them to the database itself. At present, they accept only SBML Level 2 Version 1, but say that they will soon expand to Level 1, then CellML, and eventually to other formats.

David Steven

\title{
WOUND HEALING
}

\section{Rebuilding barriers}

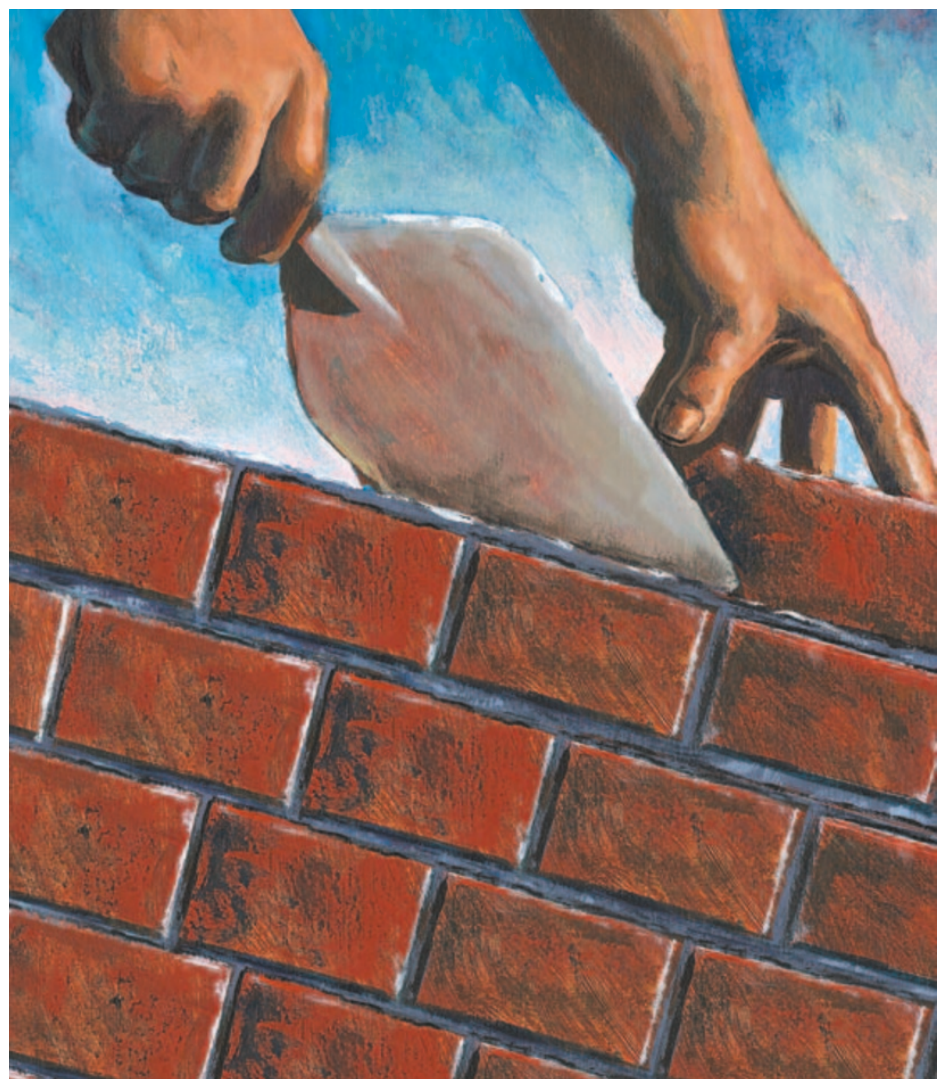

Mammals have skin; insects have cuticle - both function as barriers. It goes without saying that simple wounds in these integuments must be fixed, and William McGinnis group has defined an evolutionarily conserved pathway, involving the Grainy head (Grh) transcription factor and extracellular signal-regulated kinase (ERK), through which this is achieved.

The authors studied two genes - Ddc and ple - in Drosophila melanogaster. Ddc encodes dopa decarboxylase and ple encodes tyrosine hydroxylase; both proteins are involved in generating the integument of insects. After wounding late $D$. melanogaster embryos, $D d c$ and ple transcripts began accumulating in epidermal cells near the wound within 30 minutes, indicating that they were directly targeted by wound-induced signalling. A sequence from $-1.4 \mathrm{~kb}$ to the transcriptional start of $D d c$ was required for this response. A tagged reporter construct containing this sequence was activated in a decreasing graded response surrounding the wound, indicating that a signal produced at the injury site probably activated the wound response enhancer dose dependently.

MOLECULAR MOTORS

\section{Coordinated movement}

Microtubule-dependent molecular motors are responsible for the long-distance intracellular transport of organelles. Kinesins move organelles towards the plus end of microtubules - that is, towards the cell periphery - whereas dyneins move them towards the minus end. But how is this bidirectional transport of organelles achieved in vivo? Is there an ongoing tug-of-war or are the functions of these motors coordinated? Selvin and colleagues provide answers in Science.

They used a technique known as FIONA (fluorescence imaging with one nanometre accuracy) to observe green-fluorescentprotein-labelled peroxisomes being moved by microtubule motors inside live Drosophila melanogaster $\mathrm{S} 2$ cells. This technique provides a $1.5-\mathrm{nm}$ accuracy and a 1.1-msec time resolution (the latter is a 400-fold improvement on previous methods). They inhibited actomyosin-dependent movements to ensure that only microtubule motors were being observed, and used RNA interference to show that kinesin- 1 and the dynein heavy chain move peroxisomes in these cells. They also verified that the effects of microtubule lattice movements on peroxisome transport were minimal that is, the movements observed were related to motor-driven movement.

FIONA revealed a step size of $\sim 8 \mathrm{~nm}$ for kinesin- $\mathbf{1}$ and the dynein heavy chain, which concurs with the results of in vitro assays. In addition, the step size remained constant. This indicates that there is no tug-of-war, which would cause the step size to decrease. Instead, it seems that the motors might be turned on and off, so that they don't pull the peroxisome simultaneously. Furthermore, the speed of the in vivo peroxisome movement indicates that, at any one time, several kinesins or dyneins (up to 11 in both cases) can work together to produce speeds that are 10 times greater than those seen in vitro. The faster movements have the same step size; the steps just occur with greater rapidity.

So, how is motor coordination achieved? Answering this question 